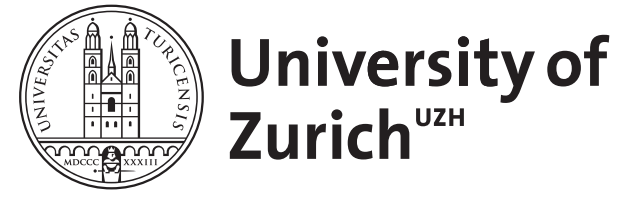

\title{
Studenten am Hausärzte-Kongress in Arosa
}

\author{
Marty, F ; Senn, O
}

\begin{abstract}
Medizinstudenten an einem Hausärztekongress? Vor 10 Jahren noch undenkbar, heute eine Realität. Dieser Beitrag schildert kurz die Entwicklung des «Studentenprojekts » am H ausärzte-Kongress Arosa und zeigt, dass ein innovatives Weiterbildungsformat auch bei angehenden Ärzten Interesse wecken kann.
\end{abstract}

Posted at the Zurich Open Repository and Archive, University of Zurich ZORA URL: https://doi.org/10.5167/uzh-96697

Journal Article

Published Version

Originally published at:

Marty, F; Senn, O (2014). Studenten am Hausärzte-Kongress in Arosa. PrimaryCare, 14(10):174. 


\section{Studenten am Hausärzte-Kongress in Arosa}

\author{
Medizinstudenten an einem Hausärztekongress? Vor \\ 10 Jahren noch undenkbar, heute eine Realität. Dieser \\ Beitrag schildert kurz die Entwicklung des «Studenten- \\ projekts» am Hausärzte-Kongress Arosa und zeigt, \\ dass ein innovatives Weiterbildungsformat auch bei \\ angehenden Ärzten Interesse wecken kann.
}

\section{Einleitung}

Der Ärztekongress Arosa ist ein «Urgestein» unter den HausärzteKongressen: Seit Mitte der 70er-Jahre treffen sich im März jeweils über 300 Hausärzte in Arosa zur Weiterbildung. Die Finanzierung, Konzeption, Organisation und Durchführung dieses Kongresses liegt bis heute in hausärztlicher Hand. Dies garantiert eine berufsnahe Weiterbildung, schlanke Strukturen und damit kurze Wege in der Vorbereitung des Kongresses und bei der Themenwahl sowie eine Offenheit gegenüber Neuem. Ein gutes Beispiel ist das sogenannte «Aroser Studentenprojekt».

\section{Die Idee}

Schon lange zeichnete sich ein Mangel an nachrückenden Hausärzten ab. Am Aroser Kongress 2006 stellten zwei initiative Kongressbesucher, Gerhard Schilling und Heinz Weber, fest, dass Hausärzte schon bei den Studenten für Nachwuchs werben müssen. Diese sollten Gelegenheit haben, sich über das «Berufsfeld Hausarzt» sowie dessen Karrieremöglichkeiten zu informieren. Der Kongressverein war über die Initiative erfreut und mit der Mepha AG, welche seit Jahren verschiedene Projekte der Hausärzte unterstützt, fand sich ein grosszügiger Sponsor: Der Kongressverein ermöglicht den kostenlosen Zugang zum Kongress und dem Workshop, Mepha offeriert den Studenten Kost und Logis.

Nur - waren Medizinstudenten für einen Hausarzt-Kongress zu begeistern? Der Einbezug von Studenten in einen fachärztlichen Kongress war Neuland!

\section{Das Projekt}

Ärzte des Schaffhauser Hausärztevereins entwickelten in der Folge ein eigenständiges Konzept, welches innerhalb des Kongresses Gastrecht erhielt. Erfahrene Hausärzte sollten die Studenten während des Kongresses betreuen und in eigens entwickelten Workshops einen Einblick in den Alltag eines Hausarztes geben. Allgemeine Informationen, Tipps zu Weiterbildungsstellen in den Spitälern und zur Praxisassistenz sowie ein attraktives Rahmenprogramm luden zum Austausch mit Jungärzten sowie älteren Kollegen ein. Im Jahre 2007 realisierten die Schaffhauser Ärzte einen ersten Versuch mit 6 Studenten. 2009 stiessen in einem zweiten Schritt Ärzte des Aargauer Hausärzteverbandes dazu, ebenfalls mit einer Gruppe von 6 Studenten. Im Jahre 2010 trat mit dem «Institut für Hausarztmedizin Bern» ein weiterer Partner mit ebenfalls 6 Studenten in das Projekt ein. Das Angebot wurde mit Informationen zum
Weiterbildungsprogramm und mit einer Börse für Weiterbildungsstellen/Praxisassistenz erweitert. Es konnte zudem ein Mitglied der Jungen Hausärzte Schweiz (JHaS) für ein Gastreferat gewonnen werden. Studenten, welche ein neues Arbeitsmodell vor Ort studieren wollten, erhielten ab 2010 Gelegenheit, auf der Hinfahrt nach Arosa das medizinische Zentrum «gleis d» beim Churer Bahnhof zu besichtigen, ein von Hausärzten iniziiertes Projekt. Im Jahre 2011 stieg die Teilnehmerzahl auf 24 Studenten.

\section{Nachhaltigkeit}

Eine Veranstaltung dieser Grösse lässt sich nicht mehr nebenbei organisieren. Es galt nun, dieses bisher eigenständige «Projekt mit Gastrecht» in den Aroser Kongress zu integrieren. Eine junge Kollegin, Nina Badertscher und ein junger Kollege, Oliver Senn, liessen sich für diese Aufgabe begeistern. Beide sind am Institut für Hausarztmedizin der Universität Zürich (IHAMZ) in der Forschung tätig, Oliver Senn ist zudem in einer Praxis in Zürich als Hausarzt tätig. Er vertritt den neuen Kongressteil im Vorstand. Es gelang so, das Angebot an die Studenten nachhaltig zu sichern.

\section{Aussicht}

In den Jahren 2013 und 2014 bewarben sich über 60 Studenten für eine Teilnahme! Das Studentenprojekt hat sich in Kürze unerwartet erfolgreich etabliert. Es ist heute fester Bestandteil des Ärztekongresses Arosa. Dieses Jahr nahmen erstmals 36 Studenten am Kongress teil. Als nächsten Schritt möchten wir mit dem Projekt noch näher ans Zielpublikum, gilt es doch, die jungen Assistenzärzte, welche sich noch nicht für eine spezifische Facharzt- Weiterbildung entschlossen haben, rechtzeitig für die Chancen einer Karriere in der Hausarztmedizin zu sensibilisieren. Daher ist eine stärkere Einbindung von Ärzten in Weiterbildung geplant.

Den Initianten, Tutoren und Instituten für Hausarztmedizin sowie der Mepha sei an dieser Stelle für ihren grossen Einsatz herzlich gedankt!

\footnotetext{
Korrespondenz

Dr. med. Franz Marty

Medizinisches Zentrum gleis d

Gürtelstrasse 46

7000 Chur

franz.marty[at]hin.ch

PD Dr. med. Oliver Senn

Institut für Hausarztmedizin

UniversitätsSpital Zürich

Pestalozzistrasse 24

8091 Zürich

oliver.senn[at]usz.ch
} 